\title{
Integration of phytochemicals and phytotherapy into cancer precision medicine
}

\author{
Thomas Efferth ${ }^{1}$, Mohamed E.M. Saeed ${ }^{1}$, Elhaj Mirghani' ${ }^{2}$ Awadh Alim², Zahir \\ Yassin $^{4}$, Elfatih Saeed ${ }^{5}$, Hassan E. Khalid ${ }^{6}$ and Salah Daak ${ }^{2,3}$ \\ ${ }^{1}$ Department of Pharmaceutical Biology, Institute of Pharmacy and Biochemistry, Johannes Gutenberg University, Mainz, \\ Germany \\ 2 Salah Wanasi Foundation for Cancer Research and Control, Khartoum, Sudan \\ ${ }^{3}$ Faculty of Medicine, International University of Africa, Khartoum, Sudan \\ ${ }^{4}$ Tayba Cancer Centre, Khartoum, Sudan \\ ${ }^{5}$ Federal Government of Sudan, Khartoum, Sudan \\ ${ }^{6}$ Department of Pharmacognosy, University of Khartoum, Khartoum, Sudan \\ Correspondence to: Thomas Efferth, email: efferth@uni-mainz.de \\ Keywords: drug resistance, network pharmacology, polypharmacology, targeted chemotherapy \\ Received: November 30,2016 Accepted: February 18,2017 Published: April 27, 2017
}

Copyright: Efferth et al. This is an open-access article distributed under the terms of the Creative Commons Attribution License 3.0 (CC BY 3.0), which permits unrestricted use, distribution, and reproduction in any medium, provided the original author and source are credited.

\section{ABSTRACT}

Concepts of individualized therapy in the 1970s and 1980s attempted to develop predictive in vitro tests for individual drug responsiveness without reaching clinical routine. Precision medicine attempts to device novel individual cancer therapy strategies. Using bioinformatics, relevant knowledge is extracted from huge data amounts. However, tumor heterogeneity challenges chemotherapy due to genetically and phenotypically different cell subpopulations, which may lead to refractory tumors. Natural products always served as vital resources for cancer therapy (e.g., Vinca alkaloids, camptothecin, paclitaxel, etc.) and are also sources for novel drugs. Targeted drugs developed to specifically address tumor-related proteins represent the basis of precision medicine. Natural products from plants represent excellent resource for targeted therapies. Phytochemicals and herbal mixtures act multispecifically, i.e. they attack multiple targets at the same time. Network pharmacology facilitates the identification of the complexity of pharmacogenomic networks and new signaling networks that are distorted in tumors. In the present review, we give a conceptual overview, how the problem of drug resistance may be approached by integrating phytochemicals and phytotherapy into academic western medicine. Modern technology platforms (e.g. "-omics" technologies, DNA/RNA sequencing, and network pharmacology) can be applied for diverse treatment modalities such as cytotoxic and targeted chemotherapy as well as phytochemicals and phytotherapy. Thereby, these technologies represent an integrative momentum to merge the best of two worlds: clinical oncology and traditional medicine. In conclusion, the integration of phytochemicals and phytotherapy into cancer precision medicine represents a valuable asset to chemically synthesized chemicals and therapeutic antibodies.

\section{INTRODUCTION}

Resistance to anticancer drugs already appeared in the very early days of cancer chemotherapy more than half a century ago [1-6], and it still hampers successful treatment of patients nowadays [7]. Many established anticancer drugs kill proliferative cells, whether or not they are malignant. This approach causes only modest tumor specificity, and non-tumorous normal proliferative tissues are also affected. Thereby, the application of drug doses high enough to kill all tumor cells including the less sensitive tumor subpopulations cannot be applied without provoking severe side effects in cancer patients. As a consequence, sub-optimal drug doses may let 
few inherently resistant tumor cells unaffected, which subsequently grow out leading to the reappearance of tumors. These refractory tumors do not respond to cytostatic therapy anymore with fatal outcomes for patients.

Current chemotherapy protocols are based on the result of prospective, randomized, double-blind phase III studies, which results in similar clinical standard treatment guidelines. However, each tumor may behave in a different manner and the treatment success of individual patients still cannot be reliably predicted, although the statistical probability of treatment response for larger groups of patients can be estimated from the results of clinical trials. The reason is that even tumors of the same origin and histology may differ from patient to patient in their biological behaviour. Even more, cells of the same tumor may be different from each there, and there is a substantial heterogeneity which greatly influences the response of tumor cell subpopulations to chemotherapy. While some subpopulations respond well to treatment, others resist and give rise to treatment failure. The appearance of drug resistance is a major, still unresolved obstacle in cancer therapy even after many decades of enormous efforts in cancer research. It has therefore been attempted to understand the molecular mechanisms of drug resistance and to predict a priori, whether or not an individual tumor would respond to drug therapy and to adapt treatment according to the individual drug sensitivity profile of tumors [8]. Whereas sensitivity or resistance to targeted drugs (e.g., HER2- or estrogen-receptortargeting small molecules or therapeutic antibodies) may be straightforward, the reliable prediction of treatment efficacy for the clinically long established cytotoxic drugs is much more complicated, since their cellular targets frequently less well-defined and these kinds of cytostatic drugs reveal broader modes of action against malignant and even normal proliferating cells.

In the present review, we give a conceptual overview, how the problem of drug resistance may be approached by integrating phytochemicals and

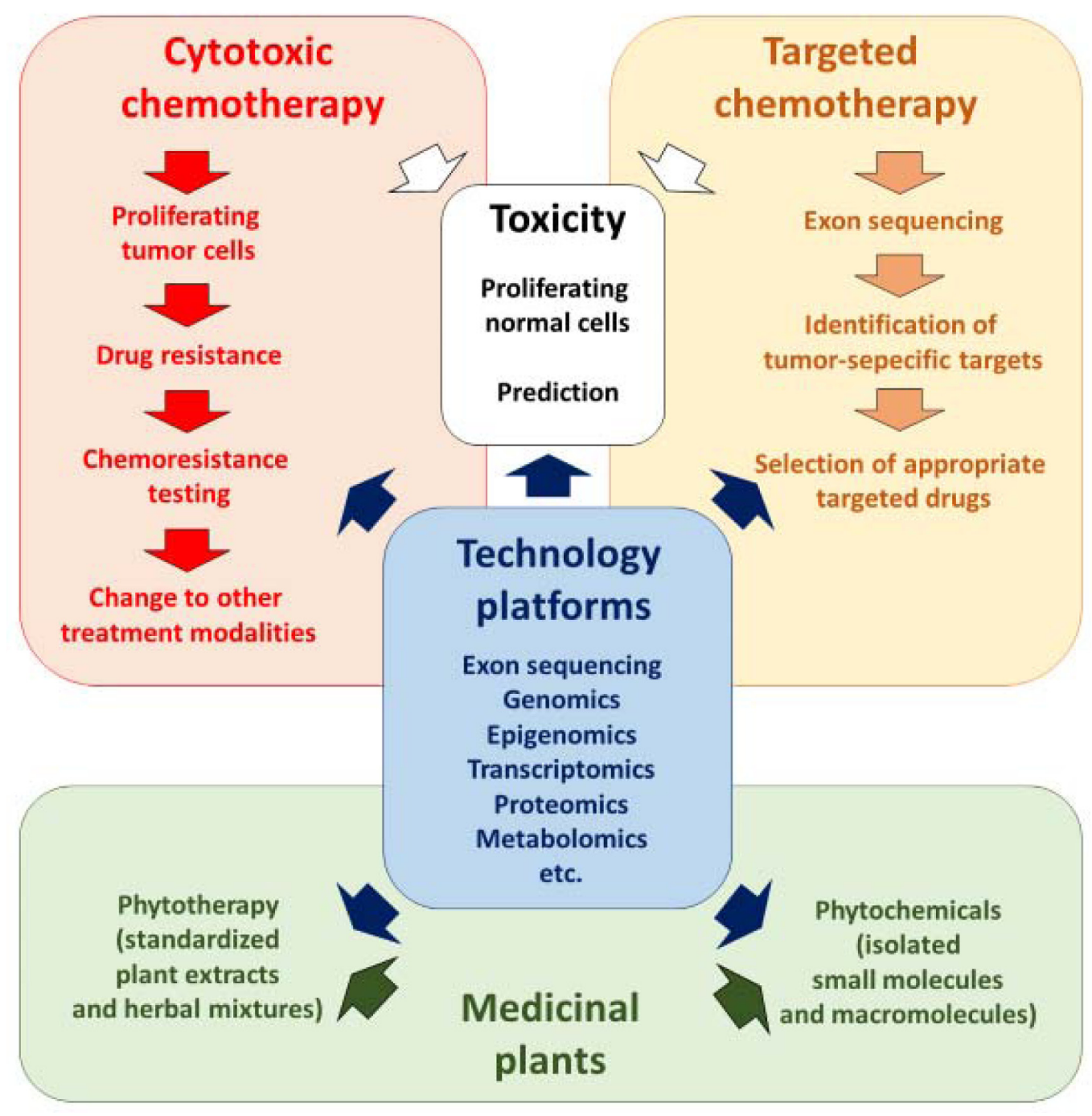

Figure 1: Integration of phytochemicals and phytotherapy into standard academic oncology. 
phytotherapy into academic western medicine. Modern technology platforms (e.g. "-omics" technologies, DNA/ RNA sequencing, and network pharmacology) can be applied for diverse treatment modalities such as cytotoxic and targeted chemotherapy as well as phytochemicals and phytotherapy (Figure 1). Thereby, these technologies represent an integrative momentum to merge the best of two worlds: clinical oncology and traditional medicine.

\section{PREDICTIONOFDRUGSENSITIVITYAND RESISTANCE}

Diagnostic tests are desirable to predict sensitivity of resistance of each individual tumor to drug treatment. If a tumor is resistant, therapy may only provoke toxicity in healthy organs without eradication the cancer itself [6]. In case a tumor is resistant to certain drugs, the treatment regimen may be changed to other still effective drugs. Therefore, early concepts of individualized therapy in the 1970s and 1980s attempted to devise diagnostic in vitro tests to predict the drug responsiveness in individual patients $[9,10]$. Although many different assays have been developed, none of them reached the status of routine clinical diagnostics [5, 11]. A meta-analysis of the literature published during the past four decades considering test results of more than 15, 000 tumor patients unambiguously demonstrated in the majority of studies that resistance was correctly predicted with accuracy between 80 and $100 \%$, while drug sensitivity could only be predicted with an accuracy of $50-80 \%$ [10]. In the past decades, the main attention of oncologist was focused on the identification of sensitive drugs which would be able to treat otherwise resistant tumors. Therefore, the high reliability of these in vitro tests to predict resistance rather than sensitivity was not sufficiently appreciated. This was mainly due to the lack of alternative treatment options in case a tumor turned out to be resistant to the available standard drugs at that time. The physicians in the clinics were understandably reluctant to tell a patient "Your tumor is predicted as being resistant. Sorry, we cannot do anything for you". Nowadays the situation has changed as more treatment strategies are available. If a tumor behaves resistant to one or several cytostatic anticancer drugs, other options may be chosen such as antibody therapy, adoptive immunotherapy, high-dose therapy, hematopoietic stem cell transplantation, supportive gene therapy protocols, hyperthermia, and also - as we will see below - phytotherapy. Therefore, the high reliability to predict resistance should be understood as a valuable chance to plan other individualized treatment options. Therefore, a rethinking of the "chemosensitivity" concept has been proposed to unravel and revive the full potential of a revival of predictive tests for personalized medicine.

The concept of predictive chemosensitivity and -resistance testing has not been documented in the literature for natural products and herbal remedies by far to the same extent as it did for standard cancer chemotherapy [12]. In traditional Chinese medicine (TCM), where individualized therapy is one of the most prominent and important features, the decision on the right combination of herbs for an individual cancer patient mainly relies on the particular TCM diagnostics rather than on predictive in vitro tests. Therefore, these kinds of assays are not very popular. In Western countries, predictive chemosensitivity assays are broadly offered by commercial sources for use in complementary and alternative medicine. Although it can well happen that they will frequently be applied, results are scarcely reported in the scientific literature.

\section{RELEVANCE OF "OMICS" TECHNOLOGIES FOR PRECISION MEDICINE AND DRUG DISCOVERY}

A crucial decision in individualized therapy is not only the choice of the right drug for the right patient, but also the right combination of drugs and the duration and dose of treatment. Because of the complexity of tumor genomes, this may be difficult to dissect with the classical methodology of clinical trials. As a direct consequence of the deciphering of the human genome at the verge from the second to the third millennium, techniques have been developed that allow to determine (1) genomic variations such as single nucleotide polymorphisms (SNPs) [13], copy number variations and other structural variations associated with disease progression and drug response (genomics), (2) epigenetic modifications such as DNAmethylation, histone acetylation or micro-RNA expression (epigenomics), (3) transcriptome-wide mRNA expression (transcriptomics), (4) proteome-wide protein and peptide expression (proteomics) in cells or tissues. These methods have frequently been termed "-omics" technologies. In recent years, the rapid technological advancements brought transcriptome-wide RNA-sequencing ("next generation sequencing") into play. The results obtained with these sophisticated techniques are evaluated by methods of computational biology and bioinformatics to extract and model the relevant knowledge gained from a vast plethora of generated data $[14,15]$. The potential of this new technological dimension of technology lies in its translation from the laboratory to practical routine for diagnosis and treatment and comprehensive approach to diagnosing tumors and tumor subtypes, to predict response to treatment and occurrence of unwanted side effects), Individualized treatment regimens may be planned based on a patient's (or tumor's) individual expression profiles to optimize the survival prognosis of cancer patients [1618]. Several conditions determine the setup of patienttailored therapies, e.g. (1) comprising meta-analyses on DNA sequencing results and related implications for drug development, and (2) the availability of individual targeted agents and biomarkers for therapy monitoring [19]. A multi-dimensional clinical genomics study of children 
and adolescent young adults with relapsed non-central nervous system solid cancers may be taken as a clue, how integrative genomic analyses and robust bioinformatics may serve to generate precision therapy protocols for the future [20].

The challenge of this new concept of precision medicine will be to delineate individual and efficient cancer therapy strategies, which are superior to traditional concepts of standardized tumor treatment. Enormous amounts of "-omics" data together with drug combination studies and biological network data analyses are required, since tumors consist of heterogeneous subpopulations with distinct biological features $[21,22]$. A pilot project in this context represents the connectivity map, which aims to establish a connection between chemicals and gene expression profiles in different cancer cell lines for more than 1700 compounds [23]. This project is certainly only a starting point, but it illustrates the complex requirements to develop individualized treatment protocols.

Advancements in molecular diagnostics is only one side of the coin, and the development of novel drugs has to keep pace, which may be an even more difficult task to master. Drug development and marketing is a time- and cost-intensive process and the number of newly approved drugs declined for decades, mainly because of their failure in clinical phase 2 trials, despite the fact that time and expenditure on drug research and development (R\&D) consistently increased during recent years [24, 25]. The hope was that the emerging high throughput technologies might supplement "-omics" technologies and next generation sequencing to generate novel drug candidates for the market.

In this context, a surprising concept emerged [26]. Existing drugs with a well-known safety and pharmacokinetic profiles that failed against certain diseases might serve as valuable drug candidates for other diseases affected by the same pathway. This phenomenon has been described as drug repositioning [27]. An intriguing example of the potential of drug repositioning is thalidomide, which has been banned as barbiturate for its teratogenic effects [28]. Later on, thalidomide has been identified as effective drug against severe erythema nodosum leprosum [29] and multiple myeloma [30]. Another less dramatic example is retinoic acid, which has been found to be active against acute promyelocytic leukemia [31].

\section{TUMOR HETEROGENEITY}

Cancers of the same histological type do not only differ from patient to patient, but also consist of heterogeneous subpopulations of cells within one and the same tumor. Heterogeneity represents a considerable challenge to cancer chemotherapy, since it aggravates the effective eradication of all cells of genetically and phenotypically different subpopulations. Even few surviving tumor cells may lead to repopulation and refractory tumors. Single-cell sequencing allows novel insights into the diversified and complex molecular architecture of heterogeneous tumors. The isolation and sequencing of single tumor cells are technically very challenging and consists of three major steps: (1) single cell isolation (e.g. by laser-capture microdissection or fluorescence-activated cell sorting), (2) whole genome amplification (e.g. with the help of Phi29 DNA polymerase), and (3) transcriptome-wide next generation sequencing technologies. The problem of tumor heterogeneity especially applies to drug resistance. Single cell sequencing will facilitate the detection of even the smallest populations of drug-resistant cells. Thereby, single-cell sequencing and may form the basis for novel and improved treatment options to eradicate drug-resistant tumor cells with specific genetic alterations [32].

\section{THE CONTRIBUTION OF NATURAL PRODUCTS}

Natural products always served as vital resources for cancer therapy. Well-known examples of the therapeutic potential of plant-derived natural products plants are the microtubule inhibiting Vinca alkaloids, the DNA topoisomerase I inhibitor camptothecin, the terpene paclitaxel, or the podophyllotoxin-derived lignans, etoposide and teniposide.

A survey of the National Cancer Institute showed that $69 \%$ of anticancer drugs approved between the $1980 \mathrm{~s}$ and 2002 are either natural products or developed based on knowledge gained from natural products [33]. Intriguingly, about three-quarters of plant-derived drugs in clinical use nowadays have their roots in traditional phytomedicines.

Although medicinal herbs belonged to the standard repertoire to combat diseases since ages, they gradually lost relevance with increasing successes of synthetic pharmaceuticals in western countries during the $20^{\text {th }}$ century. Owing to the interest in bioactive natural products as chemical lead compounds for the generation of semisynthetic derivatives with improved pharmacological features, medicinal plants as well as other natural resources (from marine or microbiological ecosystems) experience a thriving revival [34-36].

Secondary metabolites are synthesized by plants as a defense against competitors, herbivores and pathogens and as signal compounds that attract insects for reproduction. Repelling predators is crucial for plants since they do not have elaborated immunological defense mechanisms nor can they flew, because they are sessile. Secondary metabolites maintain crucial functions for survival and reproductive fitness of plants [37, 38]. In addition to toxicity, secondary plant metabolites exert pharmacological features, which make them valuable for treatment purposes. The separation of these beneficial from harmful effects is the goal of modern and pharmacognosy 
and pharmacology/toxicology [39, 40].

\section{THE CHALLENGE POSED BY PHYTOTHERAPY}

The conferment of the Nobel Award for Physiology or Medicine 2015 was Youyou Tu and her life-time achievements on artemisinin from the Chinese medicinal plant, Artemisia annua L. for malaria therapy represents and appreciation for the entire scientific community working on phytotherapy and natural products [41]. Therefore, it did not come as a surprise that the Nobel Award to Youyou $\mathrm{Tu}$ was anticipated with much enthusiasm. Now, when the celebrations are over and the grey everyday work reality is returning back, it is the right time to address the question what is coming next and how to continue from here. In other words, which sustainable actions are needed to come to long-lasting and significant improvements in TCM and phytotherapy in general for the sake of patients?

Research and development on artemisinin as malaria medication followed the rules and strategies of classical pharmacological drug, but not those of the development of a typical phytotherapeutic drug. The process ended with a chemical substance rather than with a standardized herbal product. The same applies for many other drugs which became established parts of modern pharmacopoeias. A majority of modern drugs, which are derived in one or the other way from natural sources were only inspired by nature [33], but not phytotherapies in a strict sense.

As a matter of fact, traditional medicine is being applied million times on this globe, and therefore the general conditions are basically different from those of synthetic chemical drugs. This may be an advantage, but represents a disadvantage at the same time, because there is less burning economic pressure to fulfil the strict regulations of the drug-approval authorities. If a herbal preparation is not marketed as drug by a pharmaceutical company, it can be either sold over the counter as dietary supplement without fulfilling any quality control measures, or it can be used as therapeutic drug for individual compassionate uses. These practices demonstrate that herbal medicines are popular among patients, but they frequently do not provide sufficient evidence that they are safe and efficient.

As popular herbal medicines are among physicians, practitioners and patients all over Asia, as critical they are considered among the medical community in the West. Several reasons can be discussed to explain this contradiction. One problem is certainly that herbal medicine does not belong to the standard repertoire of knowledge that is taught in our medical schools to the students. This is a fatal situation to our point of view. Another critical issue is, however, that the safety and quality of herbal products has still not been proven to the same extent, as it is routinely done for synthetic drugs.
The medical use of plants by traditional healers is frequently accompanied by spiritual and magical rituals. While these practices are doubtless of interest to cultural anthropology, rationale phytotherapy should focus solely on seeking scientific evidence for the pharmacological activity of medicinal plants. On the other hand, the demystification and "secularization" of medicinal plants also applies to industrialized countries, where "green medicine" is sometimes associated with esoteric elements.

If we consider phytotherapy as a regular discipline of life science, which rules and methods should be applied to do so? Crucial elements of quality control include [42]

- ethnobotany: documentation of traditional

knowledge;

- botanical verification of medicinal herbs (modern

taxonomy, HPLC fingerprinting, DNA sequencing);

- standardization of herbal products and mixtures;

- avoidance of contaminations and adulterations;

- elucidation of molecular and cellular modes of action;

- placebo-controlled, double-blind and randomized clinical trials.

To guarantee the marketing of safe and efficacious herbal products, national and international authorities regulate the approval of these medicines, e.g. the Food and Drug Administration (FDA) in the United States and the European Medicines Agency (EMA), both of which enforce regulatory sets of specifications including the quality, purity standards, dosage, production, precautions, storage, and labeling of these medicines [43]. Monographs provide the specifications of each plant. These monographs are usually compiled in pharmacopoeias which are considered as official documents specifying the quality, purity standards, dosage, production, precautions, storage, and labeling of medicines.

A main goal of the utilization of these diverse genetic resources is to market herbal products (i.e. bioprospecting). Since the use of medicinal plants is mainly based on the traditional knowledge of indigenous communities, commercialization of herbal products by pharmaceutical industries should, follow fair rules of benefit-sharing with those, where the knowledge comes from. Biopiracy practices by multinational companies in the past should be banned [44]. The World Health Organization (WHO) and the United Nations Educational, Scientific and Cultural Organization (UNESCO) draw the international attention on the protection of indigenous knowledge. The Nagoya protocol contains rules how to protect traditional medical knowledge and to compensate indigenous people for knowledge that is already being patented or being used in an inappropriate manner in the past. The European Parliament accepted the protocol (EU, No. 511/2014) on April 16 $6^{\text {th }} 2014$. It entered into force on October $12^{\text {th }} 2014$. Main principles are (1) informed consent of the country of origin of the resource and (2) mutually agreed terms between indigenous peoples and collaborative commercial partners. 
Summing up, the future of phytotherapy lies without doubt in the production of high-quality products, which are able to compete with synthetic drugs regarding safety and efficacy. Phytotherapy must not be mixed up with quackery practices in alternative medicine. Rationale phytotherapy should fight for its image as effective medicine with good tolerability.

\section{TARGETING TUMOR-RELATED PROTEINS WITH NATURAL PRODUCTS}

The problem with classical standard chemotherapy is that drugs do not sufficiently distinguish between normal and malignant growing cells. As long as cells are dividing, they are attacked by the drugs. A new concept is to seek for molecular differences between normal and cancerous cells and specifically attack cancer-related targets by drugs. Targeted drugs are designed to kill cancer cells by their binding to the target. Since this target is not present in normal cells, targeted cells are expected not to exert side effects on normal organs. Sophisticated techniques are used to identify targets for cancer therapy such as "-omics" technologies, cytogenetic methods, etc. Frequently, tumor cells bear amplified genes leading to protein overexpression are not present in normal cells. Also, chromosomal translocations may generate fusion genes coding for novel fusion proteins that are not existing in healthy cells. Some of these aberrantly expressed genes drive the development of cancer if they contain oncogenic sequences (driver genes). Other genetic aberrations occur in the course of tumor progression as a consequence of genomic instability of tumors. They do not have a major impact on the malignancy of cancer (passenger genes). Targeted drugs developed to specifically address proteins encoded by driver genes represent the basis for individualized or precision medicine. Instead of standardized therapy regimens for all patients suffering from the same tumor type, targeted drugs can be individually used depending on the specific expression of aberrant targets in each single patient. Two main categories of targeted drugs have been developed: (1) monoclonal antibodies that address cell-surface proteins. Examples are cetuximab and panitumumab against the epidermal growth factor receptor (EGFR), bevacizumab against vascular endothelial growth factor (VEGF) and rituximab against CD20. (2) Small chemical molecules that easily can enter tumor cells to attack intracellular targets (e.g. cancer-related kinases). Imatinib mesylate is a showcase example of historical relevance for the proof-of-principle of the entire concept of targeted therapy. This drug binds and inhibits the oncogenic BCR/ABL fusion protein. Other small molecules are erlotinib and gefitinib against EGFR, vemurafenib against BRAF and bortezomib against the proteasome.

Targeted therapy is a thriving area of cancer research, which is rapidly developing. As more novel targets will be identified by tumor DNA sequencing and other techniques, as more novel drugs may be developed to inhibit them. The scientific and economic impact of the concept of precision medicine will revolutionize cancer therapy in the years to come. Nevertheless, targeted drugs also have considerable disadvantages:

(1) Tumors frequently develop resistance against targeted drugs. Alterations in the target structure (e.g. point mutations), single nucleotide polymorphisms, cell

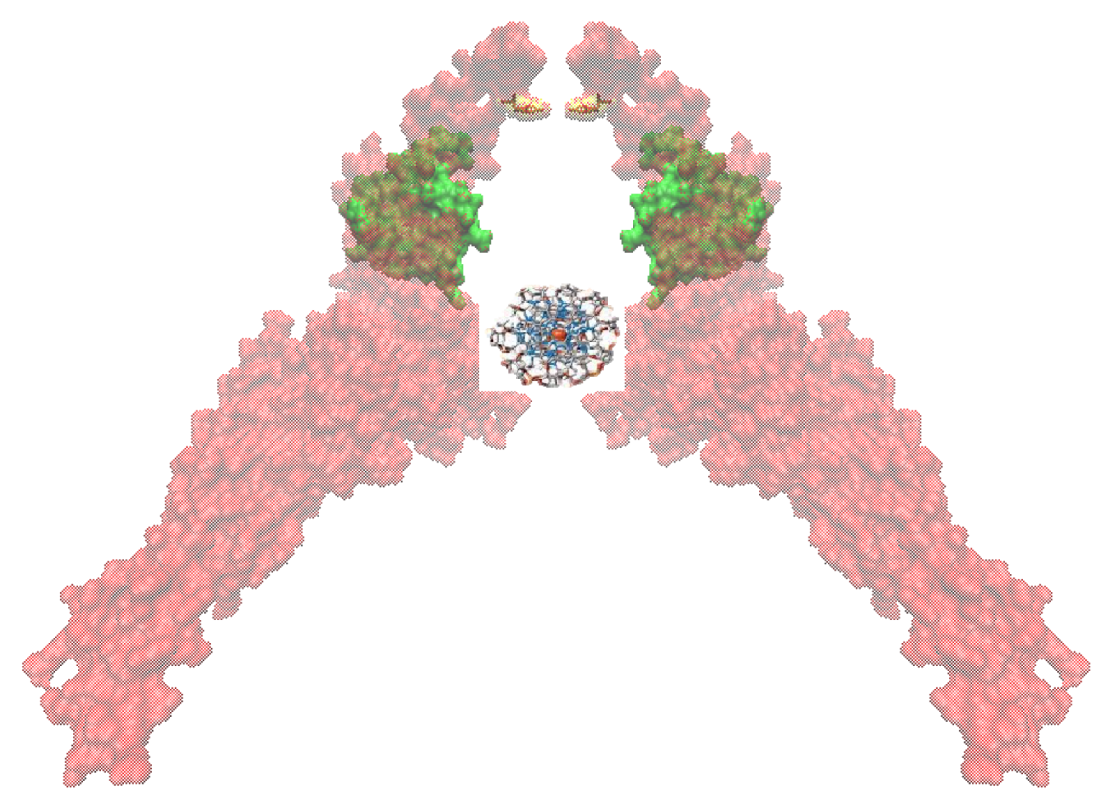

Figure 2: Homodimers of Stat3 bound to DNA for transcriptional activation. SH2 domains are shown in green and the phosphorylation sites (Tyr705) are shown in yellow representation. 
Table I: Phytochemical inhibitors of STAT3 tyrosine phosphorylation.

\begin{tabular}{|c|c|c|}
\hline Phytochemical & Plant & Reference \\
\hline Curcumin & Curcuma longa L. & Bharti et al., 2003 [120] \\
\hline Curcumin & Curcuma longa L. & Chakravarti et al., 2006 [121] \\
\hline Cryptotanshinone & Salvia miltiorhiza Bunge & Shin et al., 2009 [122] \\
\hline Cryptotanshinone & Salvia miltiorhiza Bunge & Ge et al., 2015 [123] \\
\hline Epigallocatechin-3-gallate & Camelia sinensis (L.) Kuntze & Masuda et al., 2001 [124] \\
\hline Epigallocatechin-3-gallate & Camelia sinensis (L.) Kuntze & Tang et al., 2012 [125] \\
\hline (-)epigallo-catechin gallate & Camelia sinensis (L.) Kuntze & Senggunprai et al., 2014 [126] \\
\hline Honokiol & Magnolia officinalis Rehder \& Wilson & Rajendran et al., 2012 [127] \\
\hline Honokiol & Magnolia officinalis Rehder \& Wilson & Saeed et al., 2014 [128] \\
\hline Resveratrol & various species & Bhardwaj et al., 2007 [129] \\
\hline Resveratrol & various species & Yu et al., 2008 [130] \\
\hline Cucurbitacin B & Cucumis melo L. & Yang et al., $2016[131]$ \\
\hline Cucurbitacin I & Curcurbita andreana & Blaskovich et al., 2003 [132] \\
\hline 1,2,3,4,6-penta-O-galloyl-beta-D-glucose & Paeonia suffruticosa Andrews & Lee et al., 2011 [133] \\
\hline Withacnistin & Acnistus arborescens Schltdl. & Zhang et al., 2014 [134] \\
\hline Piperlongumine & Piper longum L. & Bharadwaj et al., 2015 [135] \\
\hline Guggulsterone & Commiphora mukul, (Arn.) Bhandari & Leeman-Neill et al., 2009 [136] \\
\hline Matrine & Sophora flavescens, Aiton & Yang et al., 2015 [137] \\
\hline Eriocalyxin B & Isodon eriocalyx (Dunn) Kudo & Yu et al., 2015 [138] \\
\hline Ginkgetin & Ginkgo biloba $\mathrm{L}$. & Jeon et al., 2015 [139] \\
\hline Angoline & Zanthoxylum nitidum (Roxb.)DC & Liu et al., $2014[140]$ \\
\hline Withaferin A & Withania somnifera (L.) Dunal & Yco et al., 2014 [141] \\
\hline Chrysin & Propolis (bee glue) & $\begin{array}{l}\text { Lirdprapa-Monkol et al., } 2013 \\
\text { [142] }\end{array}$ \\
\hline Icariside II & Epimedium koreanum Nakai & Kang et al., 2012 [143] \\
\hline Licochalcone A & Glycyrrhiza inflata Batalin & $\begin{array}{l}\text { Funakoshi-Tago et al., } 2008 \\
{[144]}\end{array}$ \\
\hline Quercetin & various species & Senggunprai et al., 2014 [126] \\
\hline BP-1-102 & synthetic control compound & Zhang et al., 2012 [145] \\
\hline
\end{tabular}

cycle arrest, use of alternative signalling pathways or antigen shedding may cause ineffectiveness of treatment $[9,13]$.

(2) Unexpectedly, targeted drugs also reveal side effects in normal organs. Although the targets that are addressed by these drugs are not present in normal cells, there are nevertheless other non-specific offtarget effects. Known side effects of targeted therapies include hepatotoxicity, dermatotoxicity (skin rash, hair depigmentation, nail changes), hypertension etc.
Natural products from plants and other natural origin provide an excellent resource for targeted therapies [45-51].

(1) The number of targets for therapeutic intervention is still increasing and novel drugs for novel targets need to be developed. Natural products may serve as lead compounds that can be chemically modified to generate derivatives with improved pharmacological properties.

(2) Natural products that overcome drug resistance. 
An example is the multidrug resistance (MDR) phenotype. Drug efflux pumps of the ATP-binding cassette (ABC) transporter family confer resistance to a broad spectrum of anticancer drugs including man targeted small molecules [52]. The lignin sesamin inhibits the ATPase activity of several ABC-transporters and is thus potentially more effective in overcoming MDR than previous MDR inhibitors that block only single efflux pumps [53].

(3) The severe side effects of classical cytotoxic (non-targeted) as well as of targeted therapy may be alleviated or abolished by natural products and medicinal plants (e.g. PHY906) [54-57].

To illustrate the potential of natural products for targeted therapy, we have chosen STAT3. An important

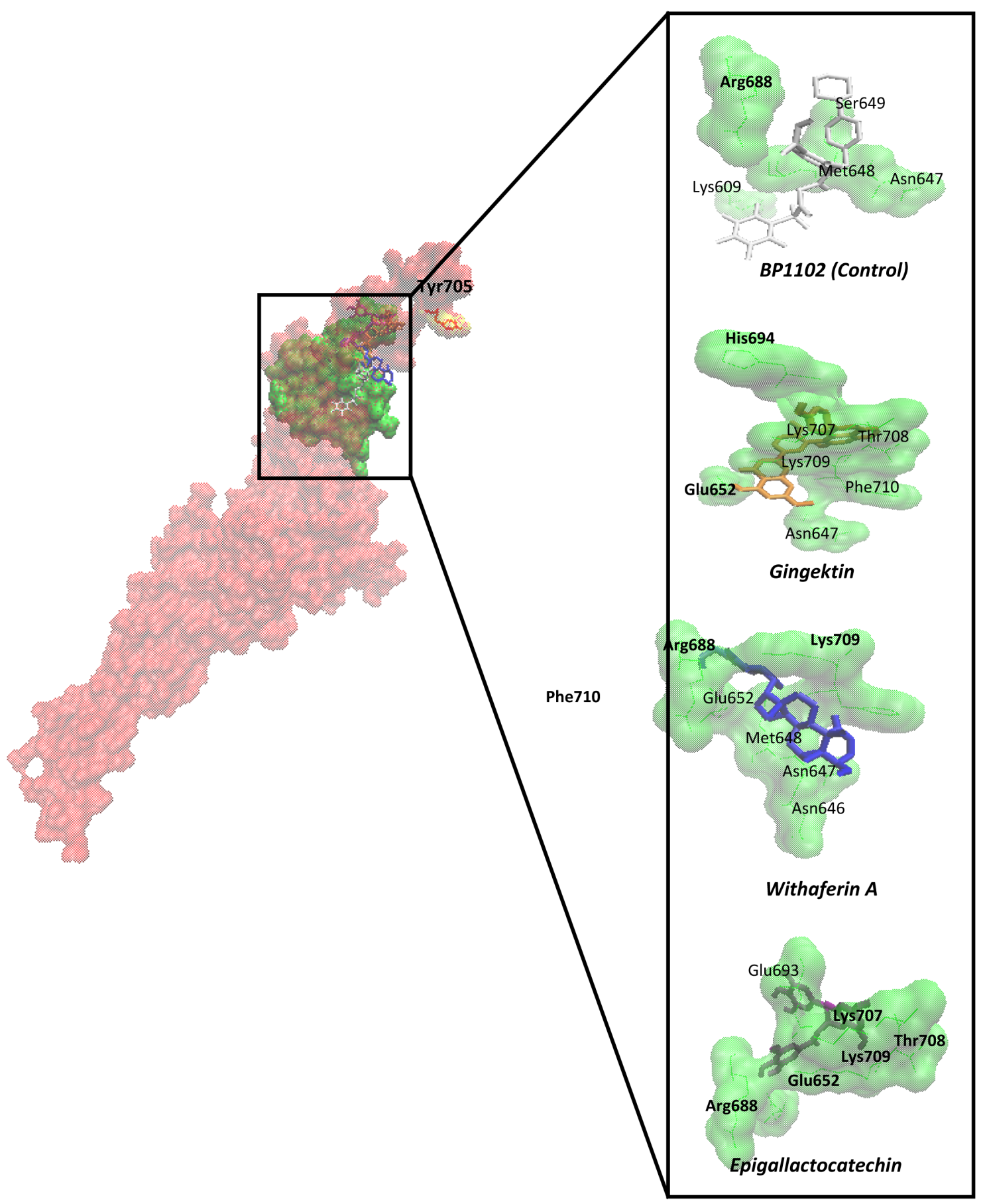

Figure 3: Defined molecular docking of phytochemicals to STAT3 at the SH2 domain. STAT3 has been represented in surface format with red and SH2 in green. Phosphorylation site was shown in yellow (Tyr705). The compounds were shown in dynamic bond format with different colors. The binding residues were visualized closely. The residues that bound to compounds by hydrogen bond were shown in bold. 
Table II: Defined molecular docking of natural products to the SH2 domain of STAT3.

\begin{tabular}{|l|c|c|}
\hline Compound & Binding Energy (kcal/mol) & $\mathbf{p K i}(\boldsymbol{\mu M})$ \\
\hline Gingektin & $-9.16 \pm 0.20$ & $0.20 \pm 0.06$ \\
\hline Withaferin A & $-8.89 \pm 0.15$ & $0.31 \pm 0.07$ \\
\hline Epigallocatechin-3-gallate & $-8.49 \pm 0.29$ & $0.65 \pm 0.27$ \\
\hline Cucurbitacin B & $-7.87 \pm 0.56$ & $0.39 \pm 0.16$ \\
\hline Cucurbitacin I & $-7.81 \pm 0.11$ & $1.90 \pm 0.38$ \\
\hline Withacnistin & $-7.54 \pm 0.09$ & $3.00 \pm 0.46$ \\
\hline BP-1-102** & $-7.46 \pm 0.36$ & $3.81 \pm 1.94$ \\
\hline Guggulsterone & $-7.29 \pm<0.001$ & $4.51 \pm 0.01$ \\
\hline Licochalcone A & $-7.02 \pm 0.06$ & $7.12 \pm 0.65$ \\
\hline Angoline & $-7.01 \pm 0.02$ & $7.25 \pm 0.24$ \\
\hline Curcumin & $-7.00 \pm 0.14$ & $7.49 \pm 1.63$ \\
\hline Piperlongumine & $-6.98 \pm 0.09$ & $7.76 \pm 1.24$ \\
\hline Neoambrosin & $-6.96 \pm 0.01$ & $7.95 \pm 0.12$ \\
\hline Damsin & $-6.83 \pm<0.001$ & $9.86 \pm 0.01$ \\
\hline Eriocalyxin B & $-6.82 \pm 0.01$ & $9.97 \pm 0.25$ \\
\hline Quercetin & $-6.63 \pm 0.04$ & $13.69 \pm 0.88$ \\
\hline Chrysin & $-6.60 \pm 0.03$ & $13.90 \pm 1.09$ \\
\hline Resveratrol & $-6.23 \pm 0.16$ & $27.76 \pm 7.96$ \\
\hline Icariside II & $-5.91 \pm 0.65$ & $33.07 \pm 16.46$ \\
\hline Matrine & $-5.86 \pm<0.001$ & $50.58 \pm 0.01$ \\
\hline Pentagalloylglucose & $-2.60 \pm 0.16$ & $12,625 \pm 3,217.34$ \\
\hline Cryptotanshinone & $-2.47 \pm 8.56$ & $3.69 \pm 0.01$ \\
\hline Lowest biningen & \\
\hline
\end{tabular}

Lowest binding energies and predicted inhibition constants (pKi) have been shown. Each docking experiment has been repeated three times.

** Synthetic drug used as control compound for comparison [131].

molecule in signal transduction processes of tumors represents signal transducer and activator of transcription 3 (STAT3). Upon binding of specific ligands (interferons, epidermal growth factor, interleukin-5 and -6) to their receptors, receptor-associated Janus kinases (JAK) are activated, which in turn activate STAT3. After phosphorylation of STAT3 at tyrosine at position 705, STAT3 dimerizes and translocates to the nucleus, where it binds to the DNA (Figure 2). As transcription factor, STAT3 induces the expression of genes involved in cell growth, apoptosis, invasion and metastatis, angiogenesis and other cellular processes. STAT3 activation can also take place by mitogen-activated protein kinases (MAPK) and c-SRC non-receptor tyrosine kinase by phosphorylation of the serine residue 727 of STAT3.

Constitutively activated STAT3 promotes carcinogenesis and STAT3 overexpression has been reported in many tumor types $[58,59]$. Recent reports indicate that STAT3 may also act as tumor suppressor depending on the mutational background [60]. There are many efforts to disrupt the STAT3 signaling route by small molecule inhibitors [61]. Three major strategies have been used to reach this goal:

(1) Inhibitors acting upstream of STAT3: Tyrosinekinase inhibitors of cell surface receptors (EGFR, HER2,
PDGFR, IGFR, etc.) inhibit downstream signalling including the STAT3 pathway. The same is true for JAK $1 / 2$, which are upstream of STAT3.

(2) STAT3 inhibitors that disrupt dimerization at the $\mathrm{SH} 2$ domain. The SH2 domain of STAT3 consists of the tyrosine-phosphorylation site at Tyr705 and two closely neighbored binding sub-pockets. Most STAT3 inhibitors bind to the $\mathrm{SH}_{2}$ domain [62].

(3) Inhibitors of the STAT3 DNA binding domain. The sterical hindrance by small molecules to bind to DNA inhibits the transcription factor activity of STAT3.

In addition to synthetic small molecules that have been described as STAT3 inhibitors, a number of phytochemicals from diverse medicinal plants also have been reported to block STAT3 phosphorylation and nuclear translocation (Table I). Molecular docking analyses demonstrate that they bind to the protein in a comparable manner as reported for synthetic small molecules (Table II). For comparison, the known STAT3 inhibitor BP-1102 was used as synthetic control compound. Its binding energy was $-7.46( \pm 0.36) \mathrm{kcal} / \mathrm{mol}$ and the $\mathrm{pKi}$ value was $3.81( \pm 1.94) \mu \mathrm{M}$ (Table II). Except of four natural products, all others revealed binding energies of lower than $-6 \mathrm{kcal} / \mathrm{mol}$. Six phytochemicals revealed binding energies even better than that of BP-1-102. The positions 
of the amino acids, which are involved in binding of the compounds to STAT3 indicate that they are located in the $\mathrm{SH}_{2}$ domain of the protein (Figure 3).

Natural products and many synthetic drugs as well are rarely mono-specific and frequently address more than one target [63]. In addition to binding to one target protein, downstream signaling is affected so that it can be assumed that rather many than single proteins or genes are involved in the modes of action of a drug. Deciphering the full complexity of cellular mechanisms and signalling pathways affected by a drug can be a tedious task.

\section{NETWORK PHARMACOLOGY WITH NATURAL PRODUCTS, MEDICINAL PLANTS AND COMPLEX HERBAL REMEDIES}

As an example to illustrate the complexity of pharmacogenomics networks, we have chosen
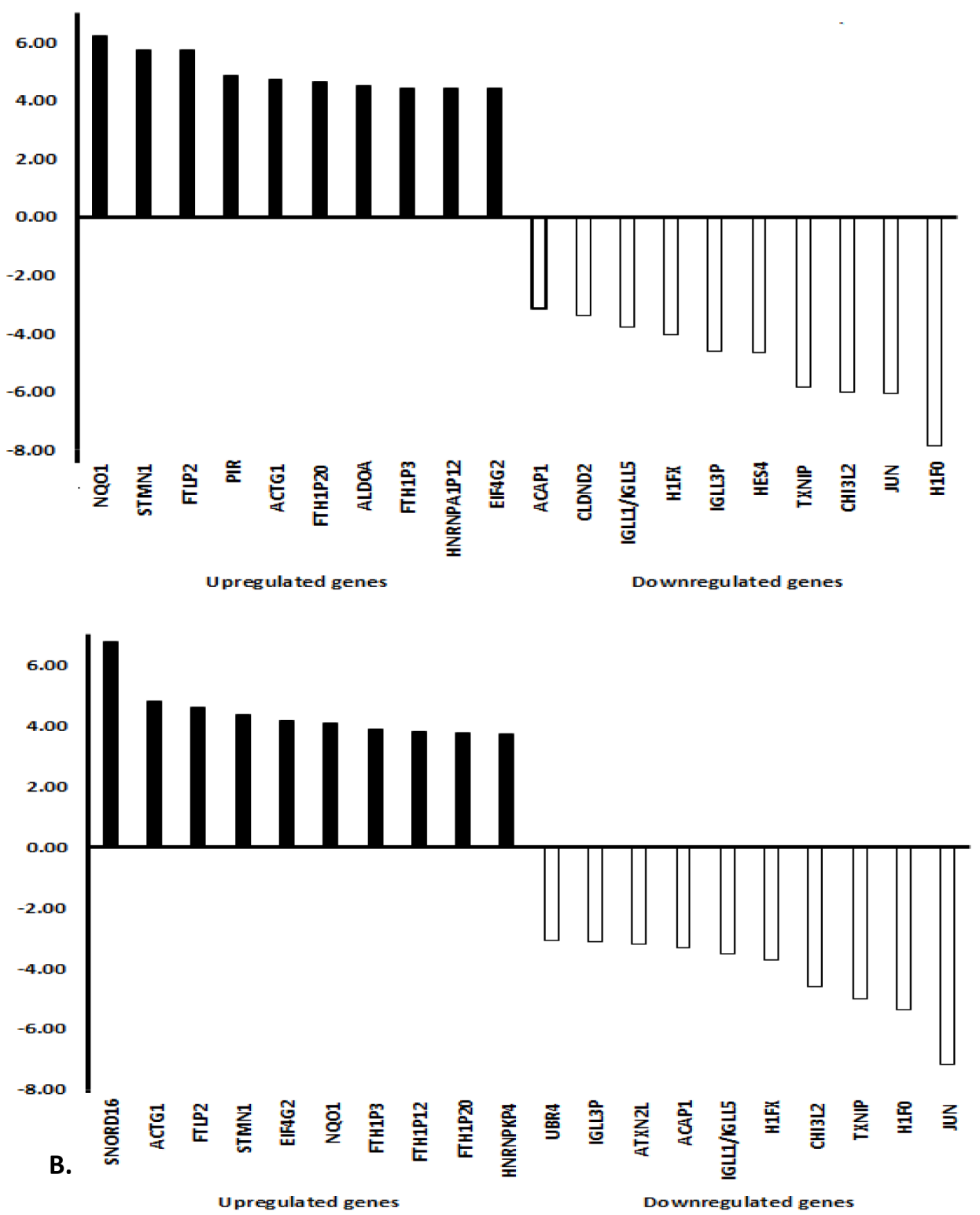

Figure 4: Top 10 increased and top 10 decreased genes in CCRF-CEM leukemia cells treated with neoambrosin or damsin from Ambrosia maritima. Leukemia cells CCRF-CEM were treated with both compounds for $24 \mathrm{~h}$. Afterwards the mRNAs were extracted and subjected to microarray hybridization on Illumina Human HT-12 Bead Chip arrays after cDNA synthesis and labelling. Microarray scanning was done using an Illumina ${ }^{\circledR}$ Bead Station array scanner (Illumina, San Diego, CA, USA) at Genomics and Proteomics Core Facility at the German Cancer Research Center (DKFZ, Heidelberg, Germany). The data were analyzed using Chipster software, subsequent assessment of significant genes was performed using empirical Bayes t-test $(\mathrm{p}<0.05)$ with Bonferroni correction. Statically significant genes were further analyzed into Ingenuity Pathway Analysis software (IPA; Ingenuity Systems, Redwood City, CA, USA) to determine cellular networks and functions affected by each compound. 
neoambrosin and damsin, two cytotoxic phytochemicals from the medicinal plant, Ambrosia maritima L. Their cytotoxic activity towards cancer cells has been reported [64]. In addition to targeting STAT3 (Table II, Figure 3, [64]), microarray analyses revealed that the expression of numerous genes was increased or decreased by these two compounds. The assignment of these genes to their corresponding signaling pathways unraveled many different actions, which all may contribute to different extent to the bioactivity of these compounds. The network analyses in Figures 4 and 5 illustrate the complex interaction networks of these two compounds. Microarray analyses revealed that the mRNA expression of 606 and 473 genes was increased or decreased after treatment of CCRF-CEM leukemia cells with neoambrosin and damsin, respectively. Figure 4 shows each the top 10 most increased or decreased genes upon treatment of cells with neoambrosin or damsin. STAT3 was also among the increased genes. These genes have been subjected to Ingenuity network analysis (Figure 5). One of the advantages of network pharmacology is that all possible actions can be identified in a comprising manner. However, at the same time this bears the danger that genes appear, which are not causatively related to the mechanism of action of a drug. The art is to separate non-relevant background noise from those signals that really contribute to the modes of action by subsequent experimentation. Genetic networks such as those shown in Figure 5 rather represent the start than the end of processes to elucidate the mode of action of drugs.

As a consequence of the complexity of drug actions, another new concept is emerging called polypharmacology, which focuses on drugs attacking multiple instead of single targets to perturb disease-associated networks $[65,66]$. To combat complex systemic diseases such as cancer, single target drugs have been proven to be less effective than promiscuous compounds that influence multiple targets and exert maximal efficacy and minimal toxicity [67-70]. The analysis of complex signaling network may unravel novel targets for drug development $[71,72]$. Network-based approaches are rapidly emerging in recent years $[65,68,73]$. Polypharmacology might also provide novel opportunities to fight drug-resistant tumor cells [74]. Network pharmacology facilitates the establishment of pragmatic network models and the prediction of drug targets based on public databases. In addition, it also helps to construct predictive 'drug target disease' network models using high-throughput screening and bioinformatics. Such approaches help to investigate underlying mechanisms of drug actions on biological networks by comparing the action of a drug with its target [75].

Cancer is a complex disease arising from changes in multiple biological networks [76], which is believed to require complex therapeutic approaches [77]. Finding drugs that act in multiple pathways, or to discern possible drug combinations represents one of the main future challenges by understanding the signaling networks of human cells and how they are altered in different cancers [78].

To be highly effective, interventions within biological networks should be multiple on the one hand, but extremely selective on the other hand to spare normal organs from detrimental side effects [79]. Holistic approaches may qualify network pharmacology as suitable tool for drug development for complex diseases such as cancer.

There are various sophisticated signaling networks driving tumorigenesis and tumor progression that can be therapeutically targeted. Instead of initial time-consuming laboratory experiments, network biology approaches for well-known pathways with various drugs might be more suitable for timely cancer drug discovery [80].

Network pharmacology facilitates the identification of new signaling networks that are distorted in various cancer types. Recently, Jaeger et al. discovered several known but unexpected as well as unknown pathways in triple negative breast cancer [81] and in ovarian carcinoma [82]. Pan-cancer network analysis of mutated networks helps to identify mutated sub-networks in cancer and paves the way to investigate new diagnostic and therapeutic prospects for specific cancer subtypes [83].

In the past few years, numerous novel signaling networks have been unraveled that contribute to carcinogenesis and tumor progression. It becomes more and more clear that the complex nature of alterations in cancer cells can hardly be attacked by drugs addressing single targets or pathways. Comprising, novel therapeutic strategies have to be devised that are able to inhibit entire malignancy-regulating networks. It is hoped that network pharmacology provides a platform for the development of a novel generation of drugs fulfilling this requirement.

Network pharmacology became a powerful tool to systematically reveal complex biological relationships. In cancer and other diseases, network pharmacology relies on "-omics" approaches to detect the variables at fundamental cellular and molecular units in response to the specific pathophysiology and/or drug treatment. The generated dataset of variables helps to generate networks from the genomic up to the metabolomic level to classify molecular processes in disease conditions. The high availability of multi-omics cancer databases opens new opportunities for data integration that promises an in-depth understanding of cancer and clinically and biologically meaningful tumor stratification [84].

The "-omics" technologies allow to measure not only well-known signaling pathways and biological networks in its entirety, but also to detect novel pathways and mechanisms that have not been described before in a given experimental context. Another considerable advantage is that cellular alterations caused by even highly complex herbal mixtures can be measured. Frequently, 

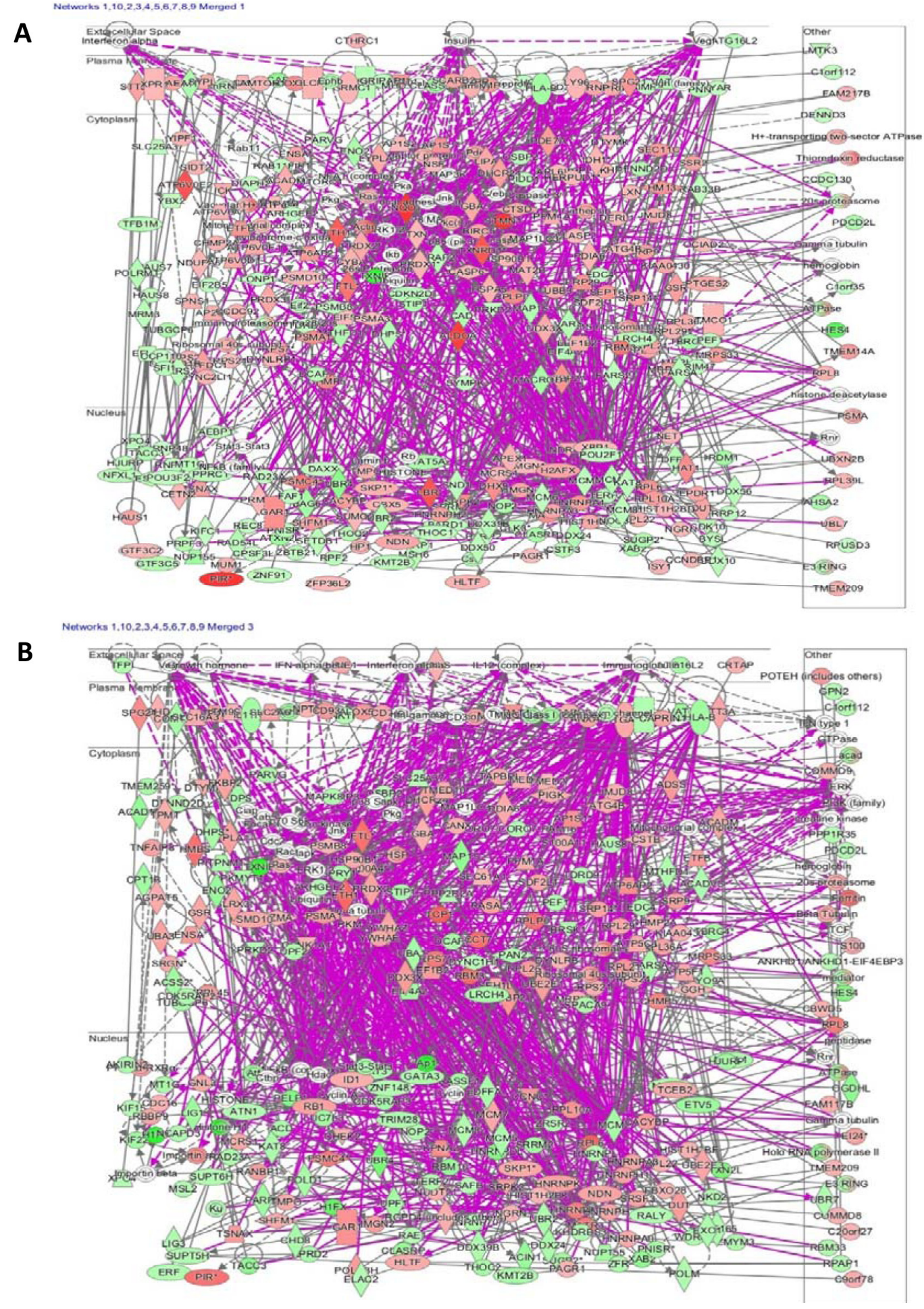

Figure 5: Top 10 out of 25 networks of CCRF-CEM leukemia cells affected by treatment with (A) neoambrosin or (B) damsin from Ambrosia maritima. Cellular molecules are represented as nodes and the biological relationship between two nodes is represented as a line. The intensity of the node color indicates the degree of up-(red) or down-(green) regulation. Solid lines show direct, dotted lines and indirect actions. Gray lines show actions within one network, purple lines show actions between different networks. The networks of neoambrosin and damsin involved RNA post-transcriptional modification, cellular assembly and organization, cellular function and maintenance, cell cycle, molecular transport, RNA trafficking, DNA replication recombination and repair, cellular growth and proliferation, as well as cell death and survival. In addition, the affected pathways for neoambrosin were NRF2-mediated oxidative stress response, EIF2 signaling, cell cycle control of chromosomal replication and protein ubiquitination pathway, whereas for damsin NRF2mediated oxidative stress response, EIF2 signaling ephrin receptor signaling, role of JAK2 in hormone-like cytokine signaling, JAK/Stat signaling were the top affected pathways. 
the holistic approach of herbal medicine cannot be satisfactorily investigated by the reductionistic methods of western science. Novel methods of network pharmacology may offer new solutions for holistic approaches.

On the other hand, some disadvantages have to be considered. With huge amounts of generated data, it may be difficult to distinguish causative mechanisms from irrelevant "background noise". Additional verification experiments are indispensable to substantiate the results obtained from "-omics" technologies. In the past, time and concentration kinetic experiments have rarely been performed in network pharmacology, because of the high costs. This bears the danger that optimal time points and drug concentrations to measure a pharmacological effect may be missed. With decreasing prices and further technology developments, this disadvantage might be overcome. Another general disadvantage is that expression analyses (e.g. transcriptomics or proteomics) do not allow to draw conclusions about the functional state of proteins (e.g. their phosphorylation state). This emphasizes the necessity to validate pathways identified by expression analyses by additional experiments.

Recently, there is a shift from microarray technologies to next generation sequencing methods such as RNA sequencing. In contrast to microarrays, this technology is not limited by the existing of sequencing information of genes. Another advantage is that background signals are much lower in RNA sequencing compared to microarray hybridization. Furthermore, the dynamic range for quantification of gene expression is much better leading to high levels of reproducibility among both technical and biological replicates.

Approaches with pleiotropic natural products that target multiple proteins and pathways in cancerassociated networks may be promising. Traditional herbal medicines play an important role in health maintenance all over the world. Herbal medicines are regarded as a valuable resource for new active compounds in drug discovery due to their multiplicity in structure, bioactivity and tolerability. The concept of 'one disease - one drug - one target' is shifting towards 'one disease - one drug multiple target' [65, 85-88]. Network-based strategies will facilitate structure-based drug design, forecast harmful side effects of drugs, and predict the effects of drugbinding on biomolecules and signaling pathways [89].

Tumors frequently develop resistance to monospecific drugs. Mutations in the corresponding target protein may easily lead to inefficacy of such a drug. By contrast, drugs addressing multiple targets are not compromised in their activity, if mutations in one of the targets appear. Most natural products exert their bioactivity by attacking multiple rather than single targets [63]. It can be speculated that during evolution of life the selective pressure favoured the emergence of multi-target specific compounds, as they make organisms more successful and competitive in the struggle for life.
Network pharmacology may provide unique opportunities for systematic target identification and possibilities to address them by multi-target specific natural compounds [90]. Highly connected nodes in complex protein networks are more vulnerable for pharmacological inhibition of the entire network than other nodes [91]. However, not all protein nodes in a network can be inhibited by drugs. It has been estimated that only about $15 \%$ of any protein nodes in a given network are druggable. To generate rational phytotherapies based on network information, several strategies can be considered:

(1) Plants or herbal mixtures can be considered, if their bioactive chemical constituents are known. This approach is based on their use in traditional medicines and is largely experience-based. In a way, herbal mixtures are comparable to multidrug combination therapy with synthetic drugs and polypharmacology [92].

(2) Multi-target specific therapy may also be reached with single phytochemicals with selective polypharmacological approaches $[93,94]$. The promiscuity of many drugs to react with more than one target has been negatively discussed in the past as off-target activities. In the context of network pharmacology, off-target effects may be reinterpreted as broader polypharmacology profiles.

(3) Recent concepts in network pharmacology emphasize the potential of synthetic lethality [95]. Proteins which a non-essential in normal cells may reach therapeutic relevance, if connected in a cancer network [96]. Their combined elimination or inhibition may lead to improved or even synergistic tumor cell eradication. Many single gene or protein knockouts exhibit no or marginal effects on tumor growth, even if relevant cancer-related targets are affected. In normal tissues, redundant protein functions and compensatory signal transduction pathways lead to robust phenotypes [97]. What makes much sense in normal physiology of organisms, poses severe treatment obstacles in cancer therapy. A conceptual solution to this problem may be not to knock out single disease-causing proteins, but to perturb entire disease-causing networks by polypharmacology with phytochemicals or complex herbal mixtures targeting multiple targets in cancer networks.

A network pharmacology approach for green tea polyphenolics revealed their multiple bioactivities towards various diseases including cancer. A total of 200 human targets were identified. This study illustrated the mechanisms of pleiotropic activity of green tea polyphenolics towards cancer, diabetes, neurodegenerative disease, cardiovascular disease, muscular disease, and inflammation [98]. Recently, a novel integrated Herbal Medicine Systems Pharmacology (HMSP) platform was used to investigate how herbs act on the human body at the molecular level. This platform supports drug absorption, distribution, metabolization and excretion (ADME) prediction, target fishing, drug target generation and data processing for the association of herbs' actions to diseases 


\section{Master Genes}

- Ligands (e.g. EGF, herceptin, WNT, chemokines,

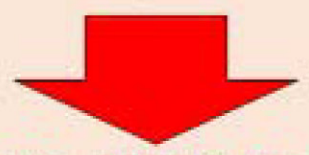

hormones etc.)

- Receptors (e.g. EGFR, HER2, Frizzled, GPCR etc.)

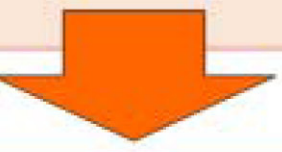

\section{Responder Genes}

- Signal transducers (e.g. JAK/STAT, RAS/RAF/ERK, PI3K/AKT, $\beta$-catenin etc.)

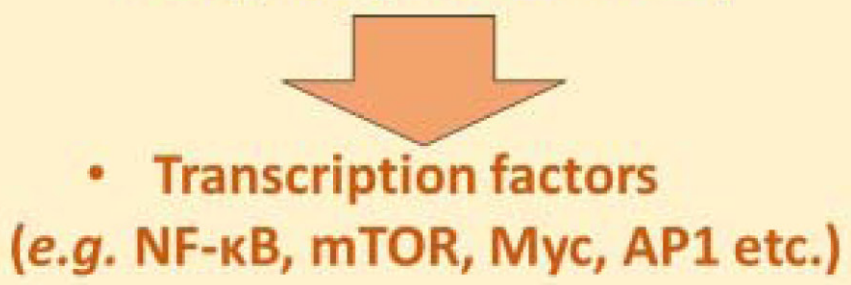

Effector Genes

- Drug resistance (e.g. ABCB1, GSTP, TOP1/2 etc.)

- Invasion, metastasis (e.g. CD44, PA MMPs etc.)

- Proliferation, cell cycle arrest (e.g. cyclins, CDKs, CKIs etc.)

- DNA repair (e.g. MGMT, XRCCs, ERCCs, MSHs, MLHs etc.)

- Angiogenesis (e.g. VEGF, ANF, TF etc.)

- Apoptosis, autophagy (e.g. BCL2, caspases, beclin etc..)

- Differentiation, tumor reversion (e.g. TCTP etc.)

Figure 6: Synopsis of main molecular mechanisms that can be targeted by synthetic small molecules, therapeutic antibodies and natural products. 
and organisms [99]. Using another systems pharmacology method, the mechanism of restoration of proper balance and harmony inside the body, organ and energy system has been elucidated for $Q i$-enriching and blood-tonifying herbs in TCM [100].

Network pharmacology may also help to better understand the reasons for failure of drugs in clinical trials regarding clinical efficacy, side effects and toxicity. The world populations are heterogeneous and genetic polymorphisms in pharmacologically relevant genes varying across geographical region are significant [101]. In addition, tumors are also heterogeneous, which is defined by presence or absence of actionable therapeutic targets [102]. The currently available therapeutic interventions for heterogeneous populations with heterogeneous tumors are still very limited. Network pharmacology approaches may overcome the pitfalls in cancer therapy and facilitate the development of novel anticancer drugs.

\section{CONCLUSIONS AND PERSPECTIVES}

The fact that chemosensitivity testing was not established as routine laboratory method may have historical reasons. In times as combination therapy protocols were established as being superior to monotherapy, data showing that tumors can reveal resistance towards many drugs at the same time was compromising the utility of drug combination regimens.

On the one hand, clinical experiences showed combination therapy protocols were able to improve treatment results, but sustainable use of patients from cancer is far from reality in many cases. On the other hand, molecular mechanisms were discovered in basic sciences that explained the appearance of broad spectrum resistance phenomena (e.g. ABC-transporter-mediated multidrug resistance, apoptosis resistance etc.). A rethinking may lead to a revival of predictive testing. Rather than prediction of drug sensitivity (which is not sufficiently reliable), drug resistance can be predicted with high precision. With multi-modal treatment options at hand, the knowledge about high probability that a specific drug would fail in a specific patient is valuable, because it allows early to switch to other more effective drugs or therapy strategies.

The development of resistance and the severe side effects of classical cytotoxic cancer therapy lead to a paradigm shift from the poorly specific cytotoxic anticancer drugs to targeted drugs, which were expected to be more tumor-specific. Indeed, this turned out to be a thriving concept with numerous new drugs on the market, which of course did not replace the classical cytotoxic drugs, but did supplement the armory to fight cancer. Although treatment outcomes could be further improved by targeted drugs they unfortunately also reveal side effects and are subject to resistance development. In this context, the therapeutic potential of phytochemicals cannot be overseen. They were already valuable in the era of cytotoxic drugs. Paclitaxel, vincristine, camptothecin, etoposide are just a few examples for established plantdrived anticancer drugs. There is a plethora of literature demonstrating that phytochemicals are also valuable for targeted cancer therapy.

Cancer stem-like cells are rare self-renewing omnipotent cells, which proliferate upon appropriate stimulation and differentiate into heterogeneous lineages in tumors. They are frequently resistant to conventional chemo- and radiotherapy. Interestingly, natural products have been described to inhibit cancer stem-like cells [103107]. This is a new field of research that is worth being investigated in more detail to understand the full range of mechanisms, which are responsible why some natural products are able to attack stem-like cells.

With the recent developments in bioinformatics, network pharmacology emerges as novel concept in therapy research. Phytotherapy with mixtures of several herbs as well as isolated single compounds exert their bioactivity by targeting multiple sites in diseased cells. A challenge for research in network pharmacology will certainly be to extract meaningful information from thousands of data points. What is a mechanistically relevant signal and what is background noise? Finding the needle in the haystack will be a task for the future and smart computer algorithms are required. Network pharmacology has to cope with multiple dimensions of problems. The multi-targeted nature of drug action, resistance development, side effects on normal organs and tissues, inter-individual biological variations, as well as inter- and intra-tumoral differences have to be considered. Especially the problem of tumor heterogeneity and possibilities to tackle with genetically diverse tumor subpopulations deserve attention from our point of view. Heterogeneous tumor populations represent a main reason for the development of resistant refractory tumors. Resistance also prevents to apply doses high enough to kill all cells, because of the severe side effects of anticancer drugs. Therefore, novel strategies to eradicate heterogeneous tumor subpopulations might not only fight the development of drug resistance but also facilitate to reduce side effects.

Integrating precision medicine into routine cancer therapy is certainly one of the predominant tasks of the next years to come (Figure 6). To realize this concept, it is not only necessary to establish the scientific basis allowing routine application in the clinic, but also to develop and integrate economic models, which allow the implementation of personalized medicine [108-111]. This is true independent of whether synthetic drugs or phytotherapeutic approaches will be used. While there is a plethora of literature on the preclinical activity of phytochemicals and medicinal plant preparations, results from clinical trials are still relatively rare. However, there are well-done clinical trials that provide evidence 
that phytochemicals and plant preparations are indeed active in the clinical setting [111-119]. For the sake of future patients, health care systems in industrialized and developing countries should do any effort to improve cure rates of tumor diseases.

\section{CONFLICT OF INTEREST}

The authors declare that there is no conflict of interest.

\section{ACKNOWLEDGMENTS}

The authors are grateful that the Salah Wanasi Foundation for Cancer Research and Control funded the publication costs of this paper. This work is dedicated to the memory of Salah Wanasi.

\section{REFERENCES}

1. Rhoads CP. Report on a cooperative study of nitrogen mustard (HN2) therapy of neoplastic disease. Transactions the the asociation of American physicians. 1947; 60:110117.

2. Pinedo HM, Giaccone G. (1998). Drug Resistance in the Treatment of Cancer. (Cambridge: Cambridge University Press).

3. Volm M, Mattern J. Resistance mechanisms and their regulation in lung cancer. Critical reviews in oncogenesis. 1996; 7:227-244.

4. Mellor HR, Callaghan R. Resistance to chemotherapy in cancer: a complex and integrated cellular response. Pharmacology. 2008; 81:275-300.

5. Mattern J, Volm M. Clinical relevance of predictive tests for cancer chemotherapy. Cancer treatment reviews. 1982; 9:267-298.

6. Lippert TH, Ruoff HJ, Volm M. Could a revision of the current guidelines for cancer drug use improve the quality of cancer treatment? Therapeutics and clinical risk management. 2014; 10:69-72.

7. Schmitt MW, Loeb LA, Salk JJ. The influence of subclonal resistance mutations on targeted cancer therapy. Nature reviews Clinical oncology. 2016; 13:335-347.

8. Walther Z, Sklar J. Molecular tumor profiling for prediction of response to anticancer therapies. Cancer journal. 2011; 17:71-79.

9. Efferth T, Konkimalla VB, Wang YF, Sauerbrey A, Meinhardt S, Zintl F, Mattern J, Volm M. Prediction of broad spectrum resistance of tumors towards anticancer drugs. Clinical cancer research. 2008; 14:2405-2412.

10. Volm M, Efferth T. Prediction of Cancer Drug Resistance and Implications for Personalized Medicine. Frontiers in oncology. 2015; 5:282.
11. Blumenthal RD, Goldenberg DM. Methods and goals for the use of in vitro and in vivo chemosensitivity testing. Molecular biotechnology. 2007; 35:185-197.

12. Wu JZ. [Tetrazolium-based colorimetric assay (MTT) and its application in chemosensitivity testing of antitumor Chinese medicine] (Article in Chinese). Zhonghua Kou Qiang Yi Xue Za Zhi. 1992; 27:373-375.

13. Efferth T, Volm M. Pharmacogenetics for individualized cancer chemotherapy. Pharmacology and therapeutics. 2005; 107:155-176.

14. Dopazo J. Genomics and transcriptomics in drug discovery. Drug discovery today. 2014; 19:126-132.

15. Feero WG, Guttmacher AE, Collins FS. Genomic medicine - an updated primer. The new England journal of medicine. 2010; 362:2001-2011.

16. Jiang L, Huang L, Kuang Q, Zhang J, Li M, Wen Z, He L. Improving the prediction of chemotherapeutic sensitivity of tumors in breast cancer via optimizing the selection of candidate genes. Computational biology and chemistry. 2014; 49:71-78.

17. Yue Z, Zhang W, Lu Y, Yang Q, Ding Q, Xia J, Chen Y. Prediction of cancer cell sensitivity to natural products based on genomic and chemical properties. Peer journal. 2015; 3:e1425.

18. Sun Y, Zhang W, Chen Y, Ma Q, Wei J, Liu Q. Identifying anti-cancer drug response related genes using an integrative analysis of transcriptomic and genomic variations with cell line-based drug perturbations. Oncotarget. 2016; 7:9404-19. doi: 10.18632/oncotarget.7012.

19. Chan SL, Wong AM, Lee K, Wong N, Chan AK. Personalized therapy for hepatocellular carcinoma: Where are we now? Cancer treatment reviews. 2016; 45:77-86.

20. Chang W, Brohl AS, Patidar R, Sindiri S, Shern JF, Wei JS, Song YK, Yohe ME, Gryder BE, Zhang S, Calzone KA, Shivaprasad N, Wen X, et al. Multi-dimensional ClinOmics for precision therapy of children and adolescent young adults with relapsed and refractory cancer: A report from the Center for Cancer Research. Clinical cancer research. 2016; 22:3810-20.

21. Gerlinger M, Rowan AJ, Horswell S, Larkin J, Endesfelder D, Gronroos E, Martinez P, Matthews N, Stewart A, Tarpey P, Varela I, Phillimore B, Begum S, et al. Intratumor heterogeneity and branched evolution revealed by multiregion sequencing. The new England journal of medicine. 2012; 366:883-892.

22. Creixell P, Schoof EM, Erler JT, Linding R. Navigating cancer network attractors for tumor-specific therapy. Nature biotechnology. 2012; 30:842-848.

23. Lamb J, Crawford ED, Peck D, Modell JW, Blat IC, Wrobel MJ, Lerner J, Brunet JP, Subramanian A, Ross KN, Reich M, Hieronymus H, Wei G, et al. The Connectivity Map: using gene-expression signatures to connect small molecules, genes, and disease. Science. 2006; 313:19291935. 
24. Mullard A. 2011 FDA drug approvals. Nature reviews in drug discovery. 2012; 11:91-94.

25. Kola I, Landis J. Can the pharmaceutical industry reduce attrition rates? Nature reviews in drug discovery. 2004; 3:711-715.

26. Aronson JK. Old drugs-new uses. British journal of clinical pharmacology. 2007; 64:563-565.

27. Ashburn TT, Thor KB. Drug repositioning: identifying and developing new uses for existing drugs. Nature reviews in drug discovery. 2004; 3:673-683.

28. Vargesson N. Thalidomide-induced teratogenesis: history and mechanisms. Birth defects research part $\mathrm{C}$, embryo today: reviews. 2015; 105:140-156.

29. Walker SL, Waters MF, Lockwood DN. The role of thalidomide in the management of erythema nodosum leprosum. Leprosy reviews. 2007; 78:197-215.

30. Moehler T. Clinical experience with thalidomide and lenalidomide in multiple myeloma. Current cancer drug targets. 2012; 12:372-390.

31. Sirota M, Dudley JT, Kim J, Chiang AP, Morgan AA, Sweet-Cordero A, Sage J, Butte AJ. Discovery and preclinical validation of drug indications using compendia of public gene expression data. Science translational medicine. 2011; 3:96ra77.

32. Schmidt F, Efferth T. Tumor Heterogeneity, Single-Cell Sequencing, and Drug Resistance. Pharmaceuticals (Basel). 2016; 9(2).

33. Newman DJ, Cragg GM. Natural products as sources of new drugs over the last 25 years. Journal of natural products. 2007; 70:461-477.

34. Efferth T. Molecular pharmacology and pharmacogenomics of artemisinin and its derivatives in cancer cells. Current drug targets. 2006; 7:407-421.

35. Kuete V, Efferth T. Cameroonian medicinal plants: Pharmacology and derived natural product. Frontiers in pharmacology. 2010; 1:123.

36. Kuete V, Efferth T. Pharmacogenomics of Cameroonian traditional herbal medicine for cancer therapy. Journal of ethnopharmacology. 2011; 137:752-766.

37. Wink M. Plant breeding: importance of plant secondary metabolites for protection against pathogens and herbivores. Theoretical and applied genetics. 1988; 75:225-233.

38. Wöll S, Kim SH, Efferth T. Animal plant warfare and secondary metabolite evolution. Natural products and bioprospecting. 2013; 3:1-7.

39. Efferth T, Miyachi H, Drexler HG, Gebhart E. Methylthioadenosine phosphorylase as target for chemoselective treatment of T-cell acute lymphoblastic leukemic cells. Blood cells, molecules and diseases. 2002; 28:47-56.

40. Li PC, Lam E, Roos WP, Zdzienicka MZ, Kaina B, Efferth T. Artesunate derived from traditional Chinese medicine induces DNA damage and repair. Cancer research. 2008; 68:4347-4351.
41. Efferth T, Zacchino S, Georgiev MI, Liu L, Wagner $\mathrm{H}$, Panossian A. Nobel Prize for artemisinin brings phytotherapy into the spotlight. Phytomedicine. 2015; 22:A1-3.

42. Efferth T, Greten HJ. Quality Control for Medicinal Plants. Medicinal and Aromatic plants (Los Angeles). 2012;1:7.

43. Efferth T, Greten HJ. The European directive on traditional herbal medicinal products: friend or foe for plant-based therapies? Zhong Xi Yi Jie He Xue Bao. 2012; 10:357-361.

44. Efferth T, Banerjee M, Paul NW, Abdelfatah S, Arend J, Elhassan G, Hamdoun S, Hamm R, Hong C, Kadioglu O, Nass J, Ochwangi D, Ooko E, et al. Biopiracy of natural products and good bioprospecting practice. Phytomedicine. 2016; 23:166-173.

45. Efferth T, Fu YJ, Zu YG, Schwarz G, Konkimalla VS, Wink M. Molecular target-guided tumor therapy with natural products derived from traditional Chinese medicine. Current medicinal chemistry. 2007; 14:2024-2032.

46. Efferth T. Personalized Cancer Medicine: From molecular diagnostics to targeted therapy with natural products. Planta medica. 2010; 76:1-12.

47. Efferth T. Signal transduction pathways of the epidermal growth factor receptor in colorectal cancer and their inhibition by small molecules. Current medicinal chemistry. 2012; 19:5735-5744.

48. Wahl O, Oswald M, Tretzel L, Herres E, Arend J, Efferth $\mathrm{T}$. Inhibition of tumor angiogenesis by antibodies, synthetic small molecules and natural products. Current medicinal chemistry. 2011; 18:3136-3155.

49. Saeed ME, Abdelgadir H, Sugimoto Y, Khalid HE, Efferth T. Cytotoxicity of 35 medicinal plants from Sudan towards sensitive and multidrug-resistant cancer cells. Journal of ethnopharmacology. 2015; 174:644-58.

50. Saeed ME, Meyer M, Hussein A, Efferth T. Cytotoxicity of South-African medicinal plants towards sensitive and multidrug-resistant cancer cells. Journal of ethnopharmacology. 2016; 186:209-23.

51. Omosa LK, Midiwo JO, Masila VM, Gisacho BM, Munayi R, Francisca-Kamakama, Chemutai KP, Elhaboob G, Saeed ME, Hamdoun S, Kuete V, Efferth T. Cytotoxicity of 91 Kenyan indigenous medicinal plants towards human CCRFCEM leukemia cells. Journal of ethnopharmacology. 2016; 179:177-96.

52. Gillet JP, Efferth T, Remacle J. Chemotherapy-induced resistance by ATP-binding cassette transporter genes. Biochimica et biophysica Acta. 2007; 1775:237-262.

53. Saeed M, Khalid H, Sugimoto Y, Efferth T. The lignan, (-)-sesamin reveals cytotoxicity toward cancer cells: pharmacogenomic determination of genes associated with sensitivity or resistance. Phytomedicine. 2014; 21:689-96.

54. Lam W, Bussom S, Guan F, Jiang Z, Zhang W, Gullen EA, Liu SH, Cheng YC. The four-herb Chinese medicine PHY906 reduces chemotherapy-induced gastrointestinal toxicity. Science translational medicine. 2010; 2:45ra59. 
55. Schröder S, Beckmann K, Franconi G, Meyer-Hamme G, Friedemann T, Greten HJ, Rostock M, Efferth T. Can medical herbs stimulate regeneration or neuroprotection and treat neuropathic pain in chemotherapy-induced peripheral neuropathy? Evidence-based complementary and alternative medicine. 2013; 2013:423713.

56. Meyer-Hamme G, Beckmann K, Radtke J, Efferth T, Greten HJ, Rostock M, Schröder S. A Survey of Chinese Medicinal Herbal Treatment for Chemotherapy-Induced Oral Mucositis. Evidence-based complementary and alternative medicine. 2013; 2013:284959.

57. Fong SY, Efferth TH, Zuo Z. Modulation of the pharmacokinetics, therapeutic and adverse effects of NSAIDs by Chinese herbal medicines. Expert opinion on drug metabolism and toxicology. 2014; 10:1711-39.

58. Kim DJ, Chan KS, Sano S, Digiovanni J. Signal transducer and activator of transcription 3 (Stat3) in epithelial carcinogenesis. Molecular carcinogenesis. 2007; 46:725-31.

59. Resemann HK, Watson CJ, Lloyd-Lewis B. The Stat3 paradox: a killer and an oncogene. Molecular and cellular endocrinology. 2014; 382:603-11.

60. Zhang HF, Lai R. STAT3 in Cancer-Friend or Foe? Cancers (Basel). 2014; 6:1408-40.

61. Furtek SL, Backos DS, Matheson CJ, Reigan P. Strategies and Approaches of Targeting STAT3 for Cancer Treatment. ACS chemical biology. 2016; 11:308-18.

62. Park IH, Li C. Characterization of molecular recognition of STAT3 SH2 domain inhibitors through molecular simulation. Journal of molecular recognition. 2011; 24:254-265.

63. Efferth T, Koch E. Complex interactions between phytochemicals. The multi-target therapeutic concept of phytotherapy. Current drug targets. 2011; 12:122-132.

64. Saeed M, Jacob S, Sandjo LP, Sugimoto Y, Khalid HE, Opatz T, Thines E, Efferth T. Cytotoxicity of the Sesquiterpene Lactones Neoambrosin and Damsin from Ambrosia maritima Against Multidrug-Resistant Cancer Cells. Frontiers in pharmacology. 2015; 6:267.

65. Hopkins AL. Network pharmacology: the next paradigm in drug discovery. Nature chemical biology. 2008; 4:682-690.

66. Xie L, Xie L, Kinnings SL, Bourne PE. Novel computational approaches to polypharmacology as a means to define responses to individual drugs. Annual review of pharmacology and toxicology. 2012; 52:361-379.

67. Azmi AS, Mohammad RM. Rectifying cancer drug discovery through network pharmacology. Future medicinal chemistry. 2014; 6:529-539.

68. Barabasi AL, Gulbahce N, Loscalzo J. Network medicine: a network-based approach to human disease. Nature reviews genetics. 2011; 12:56-68.

69. Espinoza-Fonseca LM. The benefits of the multi-target approach in drug design and discovery. Bioorganic and medicinal chemistry. 2006; 14:896-897.

70. Mencher SK, Wang LG. Promiscuous drugs compared to selective drugs (promiscuity can be a virtue). BMC clinical pharmacology. 2005; 5:3.

71. Chandra N, Padiadpu J. Network approaches to drug discovery. Expert opinion on drug discovery. 2013; 8:7-20.

72. Azmi AS, Wang Z, Philip PA, Mohammad RM, Sarkar FH. Proof of concept: network and systems biology approaches aid in the discovery of potent anticancer drug combinations. Molecular cancer therapeutics. 2010; 9:3137-3144.

73. Goh KI, Cusick ME, Valle D, Childs B, Vidal M, Barabasi AL. The human disease network. Proceedings of the National Academy of Sciences of the United States of America. 2007; 104:8685-8690.

74. Kibble M, Saarinen N, Tang J, Wennerberg K, Makela S, Aittokallio T. Network pharmacology applications to map the unexplored target space and therapeutic potential of natural products. Natural product reports. 2015; 32:12491266.

75. Zhang GB, Li QY, Chen QL, Su SB. Network pharmacology: a new approach for Chinese herbal medicine research. Evidence-based complementary and alternative medicine. 2013; 2013:621423.

76. Pache RA, Ceol A, Aloy P. NetAligner-a network alignment server to compare complexes, pathways and whole interactomes. Nucleic acids research. 2012; 40(Web Server issue):W157-161.

77. Anighoro A, Bajorath J, Rastelli G. Polypharmacology: challenges and opportunities in drug discovery. Journal of medicinal chemistry. 2014; 57:7874-7887.

78. Rask-Andersen M, Zhang J, Fabbro D, Schioth HB. Advances in kinase targeting: current clinical use and clinical trials. Trends in pharmacological sciences. 2014; 35:604-620.

79. Azmi AS. Network pharmacology for cancer drug discovery: are we there yet? Future medicinal chemistry. 2012; 4:939-941.

80. Cerami E, Demir E, Schultz N, Taylor BS, Sander C. Automated network analysis identifies core pathways in glioblastoma. PLoS one. 2010; 5:e8918.

81. Jaeger S, Min J, Nigsch F, Camargo M, Hutz J, Cornett A, Cleaver S, Buckler A, Jenkins JL. Causal Network Models for Predicting Compound Targets and Driving Pathways in Cancer. Journal of biomolecular screening. 2014; 19:791802.

82. Neapolitan R, Jiang $X$. Inferring Aberrant Signal Transduction Pathways in Ovarian Cancer from TCGA Data. Cancer informatics. 2014; 13:29-36.

83. Leiserson MD, Vandin F, Wu HT, Dobson JR, Eldridge JV, Thomas JL, Papoutsaki A, Kim Y, Niu B, McLellan M, Lawrence MS, Gonzalez-Perez A, Tamborero D, et al. Pan-cancer network analysis identifies combinations of rare somatic mutations across pathways and protein complexes. Nature genetics. 2015; 47:106-114.

84. Gevaert O, Villalobos V, Sikic BI, Plevritis SK. Identification of ovarian cancer driver genes by using 
module network integration of multi-omics data. Interface focus. 2013; 3:20130013.

85. Xu X. New concepts and approaches for drug discovery based on traditional Chinese medicine. Drug discovery today. Technologies. 2006; 3:247-253.

86. Cheung F. TCM: Made in China. Nature. 2011; 480:S82-83.

87. Azmi AS. Adopting network pharmacology for cancer drug discovery. Current drug discovery technologies. 2013; 10:95-105.

88. Li S, Fan TP, Jia W, Lu A, Zhang W. Network pharmacology in traditional Chinese medicine. Evidencebased complementary and alternative medicine. 2014; 2014:138460.

89. Engin HB, Gursoy A, Nussinov R, Keskin O. Networkbased strategies can help mono- and poly-pharmacology drug discovery: a systems biology view. Current pharmaceutical design. 2014; 20:1201-1207.

90. Korcsmaros T, Szalay MS, Bode C, Kovacs IA, Csermely P. How to design multi-target drugs. Expert opinion on drug discovery. 2007; 2:799-808.

91. Jeong H, Mason SP, Barabasi AL, Oltvai ZN. Lethality and centrality in protein networks. Nature. 2001; 411:41-42.

92. Hao da C, Xiao PG. Network pharmacology: a Rosetta Stone for traditional Chinese medicine. Drug development research. 2014; 75:299-312.

93. Morphy R, Kay C, Rankovic Z. From magic bullets to designed multiple ligands. Drug discovery today. 2004; 9:641-651.

94. Hopkins AL, Mason JS, Overington JP. Can we rationally design promiscuous drugs? Current opinion in structural biology. 2006; 16:127-136.

95. Jackson RA, Chen ES. Synthetic lethal approaches for assessing combinatorial efficacy of chemotherapeutic drugs. Pharmacology and therapeutics. 2016; 162:69-85.

96. Wang Z, Li J, Dang R, Liang L, Lin J. PhIN: A Protein Pharmacology Interaction Network Database. CPT Pharmacometrics and system pharmacology. 2015; 4:e00025.

97. Kitano H. Towards a theory of biological robustness. Molecular systems biology. 2007; 3:137.

98. Zhang S, Shan L, Li Q, Wang X, Li S, Zhang Y, Fu J, Liu X, Li H, Zhang W. Systematic Analysis of the Multiple Bioactivities of Green Tea through a Network Pharmacology Approach. Evidence-based complementary and alternative medicine. 2014; 2014:512081.

99. Luo F, Gu J, Chen L, Xu X. Systems pharmacology strategies for anticancer drug discovery based on natural products. Molecular biosystems. 2014; 10:1912-1917.

100. Liu J, Pei M, Zheng C, Li Y, Wang Y, Lu A, Yang L. A systems-pharmacology analysis of herbal medicines used in health improvement treatment: predicting potential new drugs and targets. Evidence-based complementary and alternative medicine. 2013; 2013:938764.
101. Suarez-Kurtz G, Vargens DD, Santoro AB, Hutz MH, de Moraes ME, Pena SD, Ribeiro-dos-Santos A, RomanoSilva MA, Struchiner CJ. Global pharmacogenomics: distribution of CYP3A5 polymorphisms and phenotypes in the Brazilian population. PLoS one. 2014; 9:e83472.

102. Radovich M, Clare SE, Atale R, Pardo I, Hancock BA, Solzak JP, Kassem N, Mathieson T, Storniolo AM, Rufenbarger C, Lillemoe HA, Blosser RJ, Choi MR, et al. Characterizing the heterogeneity of triple-negative breast cancers using microdissected normal ductal epithelium and RNA-sequencing. Breast cancer research and treatment. 2014; 143:57-68.

103. Efferth T. Stem cells, cancer stem-like cells, and natural products. Planta medica. 2012; 78:935-942.

104. Pistollato F, Giampieri F, Battino M. The use of plantderived bioactive compounds to target cancer stem cells and modulate tumor microenvironment. Food and chemical toxicology. 2015; 75:58-70.

105. Seo EJ, Wiench B, Hamm R, Paulsen M, Zu Y, Fu Y, Efferth T. Cytotoxicity of natural products and derivatives toward MCF-7 cell monolayers and cancer stem-like mammospheres. Phytomedicine. 2015; 22:438-443.

106. Bassa LM, Jacobs C, Gregory K, Henchey E, SerDolansky J, Schneider SS. Rhodiola crenulata induces an early estrogenic response and reduces proliferation and tumorsphere formation over time in MCF7 breast cancer cells. Phytomedicine. 2016; 23: 87-94.

107. Naveen CR, Gaikwad S, Agrawal-Rajput R. Berberine induces neuronal differentiation through inhibition of cancer stemness and epithelial-mesenchymal transition in neuroblastoma cells. Phytomedicine. 2016; 23: 736-744.

108. Atherly AJ, Camidge DR. The cost-effectiveness of screening lung cancer patients for targeted drug sensitivity markers. British journal of cancer. 2012; 106:1100-6

109. Antoñanzas F, Juárez-Castelló CA, Rodríguez-Ibeas R. Some economics on personalized and predictive medicine. The European journal of health economics. 2015; 16:985994.

110. Doble B, Tan M, Harris A, Lorgelly P. Modeling companion diagnostics in economic evaluations of targeted oncology therapies: systematic review and methodological checklist. Expert review of molecular diagnostics. 2015; 15:235-54.

111. Morgan G, Aftimos P, Awada A. Current-day precision oncology: from cancer prevention, screening, drug development, and treatment - have we fallen short of the promise? Current opinion in oncology. 2016; 28:441-6.

112. Kresty LA, Mallery SR, Stoner GD. Black raspberries in cancer clinical trials: Past, present and future. Journal of berry research. 2016; 6:251-261.

113. Weh KM, Aiyer HS, Howell AB, Kresty LA. Cranberry proanthocyanidins modulate reactive oxygen species in Barrett's and esophageal adenocarcinoma cell lines. Journal of Berry research. 2016; 6:125-136.

114 Derosa G, Romano D, D’Angelo A, Maffioli P. Berberis 
aristata/Silybum marianum fixed combination (Berberol $\left({ }^{\circledR}\right)$ ) effects on lipid profile in dyslipidemic patients intolerant to statins at high dosages: a randomized, placebo-controlled, clinical trial. Phytomedicine. 2015; 22:231-237.

115. Ebrahimpour Koujan S, Gargari BP, Mobasseri M, Valizadeh H, Asghari-Jafarabadi M. Effects of Silybum marianum (L.) Gaertn. (silymarin) extract supplementation on antioxidant status and hs-CRP in patients with type 2 diabetes mellitus: a randomized, triple-blind, placebocontrolled clinical trial. Phytomedicine. 2015;22: 290-296.

116. Mao JJ, Xie SX, Zee J, Soeller I, Li QS, Rockwell K, Amsterdam JD. Rhodiola rosea versus sertraline for major depressive disorder: A randomized placebo-controlled trial. Phytomedicine. 2015; 22:394-399.

117. Lin Y, Kazlova V, Ramakrishnan S, Murray MA, Fast D, Chandra A, Gellenbeck KW. Bone health nutraceuticals alter microarray mRNA gene expression: A randomized, parallel, open-label clinical study. Phytomedicine. 2016; 23: $18-26$.

118. Maulik SK, Wilson V, Seth S, Bhargava B, Dua P, Ramakrishnan S, Katiyar CK. Clinical efficacy of water extract of stem bark of Terminalia arjuna (Roxb. ex DC.) Wight \& Arn. in patients of chronic heart failure: a doubleblind, randomized controlled trial. Phytomedicine. 2016; 23: 1211-1219.

119. Mao JJ, Xie SX, Keefe JR, Soeller I, Li QS, Amsterdam JD. Long-term chamomile (Matricaria chamomilla L.) treatment for generalized anxiety disorder: A randomized clinical trial. Phytomedicine. 2016; 23: 1735-1742.

120. Bharti AC, Donato N, Aggarwal BB. Curcumin (diferuloylmethane) inhibits constitutive and IL-6-inducible STAT3 phosphorylation in human multiple myeloma cells. Journal of immunology. 2003; 171:3863-3871.

121. Chakravarti N, Myers JN, Aggarwal BB. Targeting constitutive and interleukin-6-inducible signal transducers and activators of transcription 3 pathway in head and neck squamous cell carcinoma cells by curcumin (diferuloylmethane). International journal of cancer. 2006; 119:1268-1275.

122. Shin DS, Kim HN, Shin KD, Yoon YJ, Kim SJ, Han DC, Kwon BM. Cryptotanshinone inhibits constitutive signal transducer and activator of transcription 3 function through blocking the dimerization in DU145 prostate cancer cells. Cancer research. 2009; 69:193-202.

123. Ge Y, Yang B, Xu X, Dai Q, Chen Z, Cheng R. Cryptotanshinone acts synergistically with imatinib to induce apoptosis of human chronic myeloid leukemia cells. Leukemia and lymphoma. 2015; 56:730-738.

124. Masuda M, Suzui M, Weinstein IB. Effects of epigallocatechin-3-gallate on growth, epidermal growth factor receptor signaling pathways, gene expression, and chemosensitivity in human head and neck squamous cell carcinoma cell lines. Clinical cancer research. 2001; 7:4220-4229.
125. Tang SN, Fu J, Shankar S, Srivastava RK. EGCG enhances the therapeutic potential of gemcitabine and CP690550 by inhibiting STAT3 signaling pathway in human pancreatic cancer. PLoS one. 2012; 7:e31067.

126. Senggunprai L, Kukongviriyapan V, Prawan A, Kukongviriyapan U. Quercetin, EGCG exhibit chemopreventive effects in cholangiocarcinoma cells via suppression of JAK/STAT signaling pathway. Phytotherapy research. 2014; 28:841-848.

127. Rajendran P, Li F, Shanmugam MK, Vali S, Abbasi T, Kapoor S, Ahn KS, Kumar AP, Sethi G. Honokiol inhibits signal transducer and activator of transcription-3 signaling, proliferation, and survival of hepatocellular carcinoma cells via the protein tyrosine phosphatase SHP-1. Journal of cellular physiology. 2012; 227:2184-2195.

128. Saeed M, Kuete V, Kadioglu O, Bortzler J, Khalid H, Greten HJ, Efferth T. Cytotoxicity of the bisphenolic honokiol from Magnolia officinalis against multiple drugresistant tumor cells as determined by pharmacogenomics and molecular docking. Phytomedicine. 2014; 21:15251533.

129. Bhardwaj A, Sethi G, Vadhan-Raj S, Bueso-Ramos C, Takada Y, Gaur U, Nair AS, Shishodia S, Aggarwal BB. Resveratrol inhibits proliferation, induces apoptosis, and overcomes chemoresistance through down-regulation of STAT3 and nuclear factor-kappaB-regulated antiapoptotic and cell survival gene products in human multiple myeloma cells. Blood. 2007; 109:2293-2302.

130. Yu LJ, Wu ML, Li H, Chen XY, Wang Q, Sun Y, Kong QY, Liu J. Inhibition of STAT3 expression and signaling in resveratrol-differentiated medulloblastoma cells. Neoplasia. 2008; 10:736-744.

131. Yang T, Liu J, Yang M, Huang N, Zhong Y, Zeng T, Wei R, Wu Z, Xiao C, Cao X, Li M, Li L, Han B, Yu X, et al. Cucurbitacin $\mathrm{B}$ exerts anti-cancer activities in human multiple myeloma cells in vitro and in vivo by modulating multiple cellular pathways. Oncotarget. 2017;8:5800-5813. doi: 10.18632/oncotarget.10584.

132. Blaskovich MA, Sun J, Cantor A, Turkson J, Jove R, Sebti SM. Discovery of JSI-124 (cucurbitacin I), a selective Janus kinase/signal transducer and activator of transcription 3 signaling pathway inhibitor with potent antitumor activity against human and murine cancer cells in mice. Cancer research. 2003; 63:1270-1279.

133. Lee HJ, Seo NJ, Jeong SJ, Park Y, Jung DB, Koh W, Lee EO, Ahn KS, Lu J, Kim SH. Oral administration of penta-O-galloyl-beta-D-glucose suppresses triple-negative breast cancer xenograft growth and metastasis in strong association with JAK1-STAT3 inhibition. Carcinogenesis. 2011; 32:804-811.

134. Zhang X, Blaskovich MA, Forinash KD, Sebti SM. Withacnistin inhibits recruitment of STAT3 and STAT5 to growth factor and cytokine receptors and induces regression of breast tumours. British journal of cancer. 2014; 111:894902. 
135. Bharadwaj U, Eckols TK, Kolosov M, Kasembeli MM, Adam A, Torres D, Zhang X, Dobrolecki LE, Wei W, Lewis MT, Dave B, Chang JC, Landis MD, et al. Drugrepositioning screening identified piperlongumine as a direct STAT3 inhibitor with potent activity against breast cancer. Oncogene. 2015; 34:1341-1353.

136. Leeman-Neill RJ, Wheeler SE, Singh SV, Thomas SM, Seethala RR, Neill DB, Panahandeh MC, Hahm ER, Joyce SC, Sen M, Cai Q, Freilino ML, Li C, et al. Guggulsterone enhances head and neck cancer therapies via inhibition of signal transducer and activator of transcription-3. Carcinogenesis. 2009; 30:1848-1856.

137. Yang N, Han F, Cui H, Huang J, Wang T, Zhou Y, Zhou J. Matrine suppresses proliferation and induces apoptosis in human cholangiocarcinoma cells through suppression of JAK2/STAT3 signaling. Pharmacology reports. 2015; 67:388-393.

138. Yu X, He L, Cao P, Yu Q. Eriocalyxin B Inhibits STAT3 Signaling by Covalently Targeting STAT3 and Blocking Phosphorylation and Activation of STAT3. PLoS one. 2015; 10:e128406.

139. Jeon YJ, Jung SN, Yun J, Lee CW, Choi J, Lee YJ, Han DC, Kwon BM. Ginkgetin inhibits the growth of DU-145 prostate cancer cells through inhibition of signal transducer and activator of transcription 3 activity. Cancer science. 2015; 106:413-420.

140. Liu J, Zhang Q, Ye Y, Li W, Qiu J, Zhan R, Chen W, $\mathrm{Yu}$ Q. Angoline: a selective IL-6/STAT3 signaling pathway inhibitor isolated from Zanthoxylum nitidum. Phytomedicine. 2014; 21:1088-1091.
141. Yco LP, Mocz G, Opoku-Ansah J, Bachmann AS. Withaferin A Inhibits STAT3 and Induces Tumor Cell Death in Neuroblastoma and Multiple Myeloma. Biochemistry insights. 2014; 7:1-13.

142. Lirdprapamongkol K, Sakurai H, Abdelhamed S, Yokoyama S, Athikomkulchai S, Viriyaroj A, Awale S, Ruchirawat S, Svasti J, Saiki I. Chrysin overcomes TRAIL resistance of cancer cells through Mcl-1 downregulation by inhibiting STAT3 phosphorylation. International journal of oncology. 2013; 43:329-337.

143. Kang SH, Jeong SJ, Kim SH, Kim JH, Jung JH, Koh W, Kim DK, Chen CY. Icariside II induces apoptosis in U937 acute myeloid leukemia cells: role of inactivation of STAT3-related signaling. PLoS one. 2012; 7:e28706.

144. Funakoshi-Tago M, Tago K, Nishizawa C, Takahashi K, Mashino T, Iwata S, Inoue H, Sonoda Y, Kasahara T. Licochalcone A is a potent inhibitor of TEL-Jak2-mediated transformation through the specific inhibition of Stat3 activation. Biochemical pharmacology. 2008; 76:16811693.

145. Zhang X, Yue P, Page BD, Li T, Zhao W, Namanja AT, Paladino D, Zhao J, Chen Y, Gunning PT, Turkson J. Orally bioavailable small-molecule inhibitor of transcription factor Stat3 regresses human breast and lung cancer xenografts. Proceedings of the National Academy of Sciences of the United States of America. 2012; 109:9623-9628. 